\title{
Applicability of Phased Array Antenna to Ground Penetrating Radar
}

\author{
Kaito KIKUCHI ${ }^{1}$, Hitoshi MIKADA ${ }^{1}$ and Junichi TAKEKAWA ${ }^{1}$ \\ ${ }^{1}$ Dept. of Civil and Earth Res. Eng., Kyoto University
}

\begin{abstract}
Ground Penetrating Radar (GPR) has been widely used to detect objects in shallow subsurface. Measuring the reflected electromagnetic (EM) waves from the subsurface, mines, remains or buried pipes can be detected by GPR. However, there is one problem in the current practice of GPR surveys. No subsurface image could be obtained for subsurface beneath surface structures since no survey lines could be drawn above the structures. To overcome this problem, we propose the use of phased array antenna as a radar source.

We conducted some numerical simulations using a 3D-FDTD method and examined the performance of the phased array antenna in terms of signal-to-noise ratio of the generated EM wave field and the response to slanted EM wave field.

Our results indicate that the application of the phased array antenna to GPR could enhance the signal-to-noise ratio for reflectors located lateral to the survey line. Also, we conclude that our proposing GPR system has a potential to be used as an angular-scanner imaging tool that has not been attempted in the conventional GPR system.
\end{abstract}

\section{INTRODUCTION}

GPR is a non-destructive tool commonly used for detecting objects in shallow subsurface such as buried pipes, land mines or tree roots ${ }^{1)}$. We measure EM waves scattered by anomalies in the subsurface in response to an EM plane wave generated by the radar. Analyzing the obtained data, we can estimate the location of anomalies. However, artificial structures on the ground often limit the extent of the survey areas. The image of subsurface structure beneath artificial structures cannot be obtained since no survey line can be drawn above the structures.

In this study, we propose the use of a phased array system ${ }^{2)}$ to overcome this problem. A phased array system is used for an air radar system. Using this system, we achieve a high directivity in the EM wave fields ${ }^{3)}$. Underground structure is normally inhomogeneous which may cause more complex wave phenomena than the air such as scattering. This means that understanding the behavior of direction-controlled EM waves in inhomogeneous medium is essential when we use the phased array system in the subsurface.

We conducted some numerical simulations using a 3-D FDTD method to understand the radiation pattern of the time shifted EM waves. Firstly, we describe the theory of numerical method and our strategy of generating slanted EM waves. Then we apply these theories to GPR. After confirming the propagation of EM wave field in the subsurface, we compare a dipole antenna and a phased array antenna. Finally, we move the phased array antenna assuming an actual GPR survey.

\section{THEORY}

\section{(1) FDTD Method}

EM fields in a linear, isotropic medium are described from Maxwell's equations ${ }^{4)}$

$$
\begin{gathered}
\operatorname{rot} \boldsymbol{E}=-\mu \frac{\partial \boldsymbol{H}}{\partial t} \\
\operatorname{rot} \boldsymbol{H}=\varepsilon \frac{\partial \boldsymbol{E}}{\partial t}+\sigma \boldsymbol{E} \\
\operatorname{div} \boldsymbol{E}=0 \\
\operatorname{div} \boldsymbol{H}=0
\end{gathered}
$$

where $\boldsymbol{E}$ and $\boldsymbol{H}$ are electric and magnetic vector fields, respectively. $\varepsilon, \mu$ and $\sigma$ are the electric permittivity, the magnetic permeability and electric conductivity of the medium, respectively.

We adopt Yee's staggered grid $^{5)}$ to calculate the $\boldsymbol{E}$ and $\boldsymbol{H}$ fields so as to simulate how EM wave 
fields propagate in the model using a 3-D FDTD method. C-PML absorbing condition ${ }^{6)}$ is applied in this simulation to avoid reflections from ground model edges.

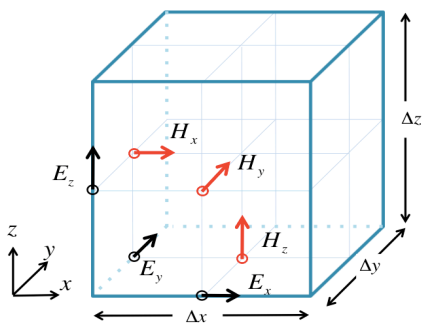

Figure 1: Yee's staggered grid

\section{(2) Antenna Design}

To confirm the applicability of phased array antenna to GPR, we compare two kinds of antenna, a dipole antenna and a phased array antenna. The phased array antenna has an $8 \times 8$ units (Figure 2, right) and both antennas have the length of about 0.5 meters.

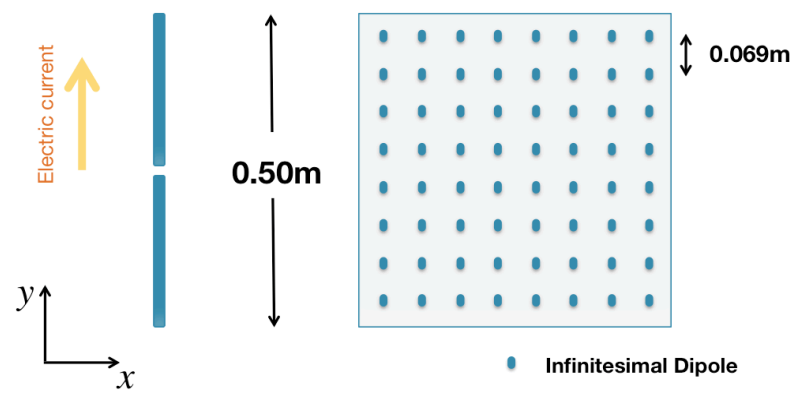

Figure 2: Dipole Antenna and Phased Array Antenna

\section{(3) Input Signal}

The electrical current source we use is the normalized 1st derivative of a Gaussian pulse (Figure 3). The frequency spectrum of the input signal is obtained by applying discrete Fourier Transform to the signal (Figure 4).

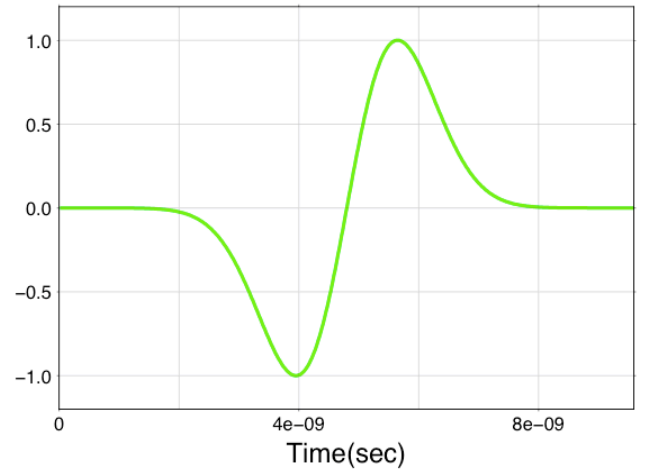

Figure 3: Input signal is 1st derivative of a Gaussian pulse

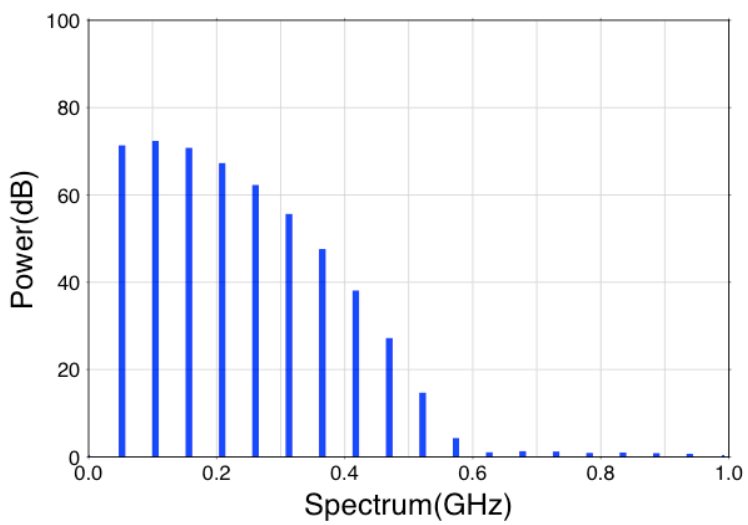

Figure 4: Frequency spectrum of the input signal

\section{(4) Phase Shifting Theory}

To direct the energy towards a desired direction in the ground, we control the time delay of each antenna of the array. Let us suppose the 8 antennas in the same $y$-coordinate (the smallest $y$ ) are $y 1$ gather. Assuming every 8 sets of antennas as $y 2, y 3$,

*.. $y 8$ (Figure 5).

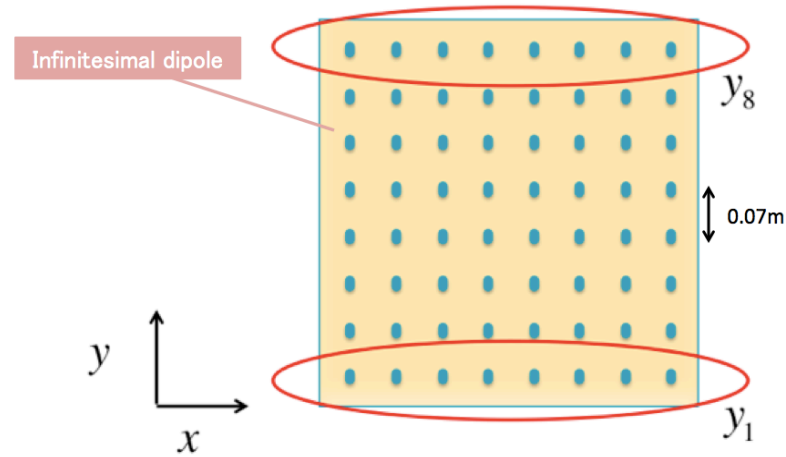

Figure 5: Antenna gathers

The time delay we give to $y \mathrm{n}$ is described as

$$
\Delta t \mathrm{n}=(n-4.5) \sin \theta / v \quad(n=1,2, \cdots, 8)
$$

where $d$ is the interval of each antenna and $v$ is the velocity of the EM waves in the dry sand. $\theta$ represents the deviation from the vertical direction.

\section{(5) Kirchhoff Migration}

To estimate the position of scatterings, we use the Kirchhoff migration method. The basic idea of the method is to back-propagate the observed data, using the equation below which is derived by applying an integral solution method to the scalar wave equation. 


$$
\begin{gathered}
U(\boldsymbol{r})=\frac{1}{2 \pi} \frac{\cos \theta}{r V}\left(\frac{\partial}{\partial t} U\left(\boldsymbol{r}_{0}, t_{0}\right)-\frac{V}{r} U\left(\boldsymbol{r}_{0}, t_{0}\right)\right) \\
t_{0}=t-\frac{r}{V} \\
r=\left|\boldsymbol{r}-\boldsymbol{r}_{0}\right|
\end{gathered}
$$

where $U\left(\boldsymbol{r}_{0}, t_{0}\right)$ is the observed data on the surface and $U(\boldsymbol{r})$ is the wave field at $\boldsymbol{r}$.

\section{Model Setup}

We conducted some numerical simulations using a 3-D ground model, which includes 100 water bodies and 100 perfect electric conductor (PEC) bodies with cubic shape which work as obstacles. The background medium is assumed to dry sand and the objects we try to detect by GPR are the concrete cubes lying at the bottom of the model (Figure 6).

In our experiments, the size of the concrete cubes is smaller than the prominent wavelength in order to observe the Rayleigh scattering.

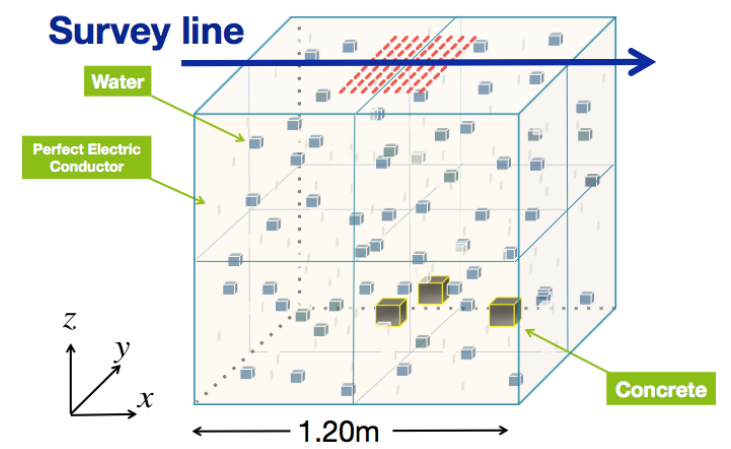

Figure 6: 3-D ground model. Blue cubes represent the water bodies and white cubes represent the PEC bodies. The blown cubes are the concrete bodies.

\section{Numerical Simulations and Results}

First, we confirmed the behavior of the slanted EM wave field in the subsurface. The phased array antenna is located at the top of the model (Figure 7).

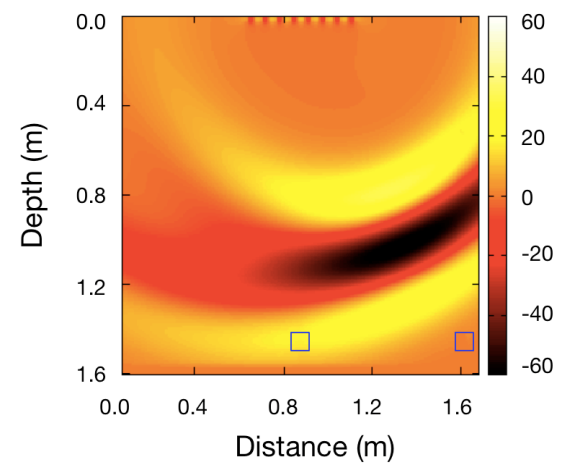

Figure 7: Slanted EM wave field in the subsurface.

We confirmed that the EM wave fields propagate diagonally downwards in the inhomogeneous ground model.

Next, we removed the two concrete cubes along the survey line and leave just one cube lying lateral to the survey line. Then, we compared the property of a dipole antenna and a phased array antenna. Both antennas are fixed and the phased array antenna radiates the energy towards a concrete cube.

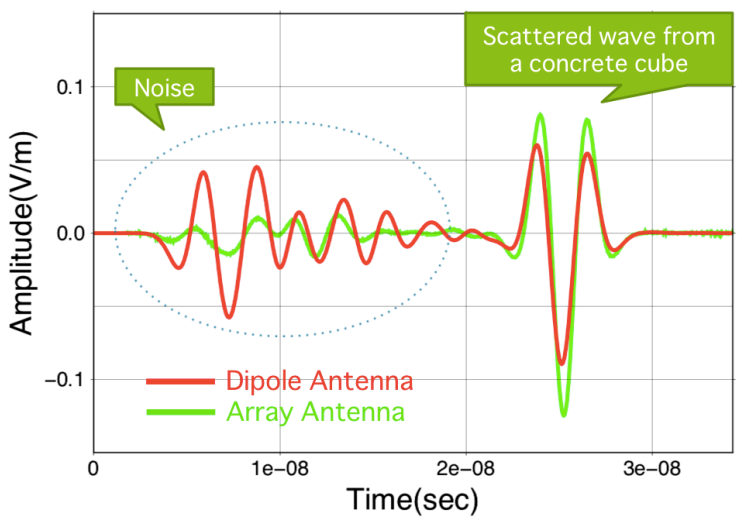

Figure 8: Comparison of scattered waves of dipole antenna and array antenna.

Figure 8 shows the scattered waves observed by a receiver at the centre of the radar. Red and green lines show the waveforms obtained by the dipole and the phased array antenna, respectively.

The continuous vibrations between $0(\mathrm{sec})$ and $2.0 \mathrm{e}-08(\mathrm{sec})$ represent the scattered waves from the obstacles. The stronger wave after the vibrations represents the scattered wave from a concrete cube. Comparing red and green waveforms, we conclud that the phased array antenna has an advantage in enhancing the amplitude of a scattered wave from the concrete cube located out of the survey line and also enhancing the signal-to-noise ratio. This results from random overlaps of 64 signals of each antenna of the array. Since the transmitting units are arranged planarly, each transmitting signal of the 
phased array is more random than the dipole.

After confirming the advantages of phased array antenna, two cubes of concrete are rearranged (Figure 6). In this model, two cubes along the survey line and one cube out of the survey line. We move the phased array antenna along the line and conducted numerical simulations at 32 source positions. In this case, the array antenna radiates the energy towards the cube out of the survey line. To estimate the position of a concrete cube, we apply Kirchhoff migration to the observed data and visualize the subsurface on $x z$-plane which includes the cube out of the survey line.

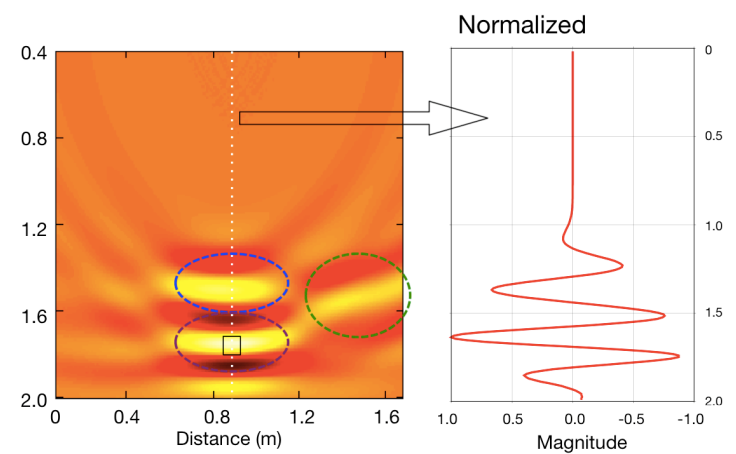

Figure 9: Subsurface image after applying migration

The strong signal stands for the estimated position of the concrete cubes (Figure 9, left). In this image, the positions of cube seem to be properly described (purple dotted circle). However, there seems to be some undesired signals remained (blue and green dotted circles). These signals are resulting from the scattered waves of concrete cubes along the survey line. The red line (Figure 9, right) represents the waveform along the white dotted line in the picture (Figure 9, left).

Finally, we use every units as a transmitter and a receiver and we stack the obtained signals in order to achieve the directivity not only in the phase of transmitting but also receiving.

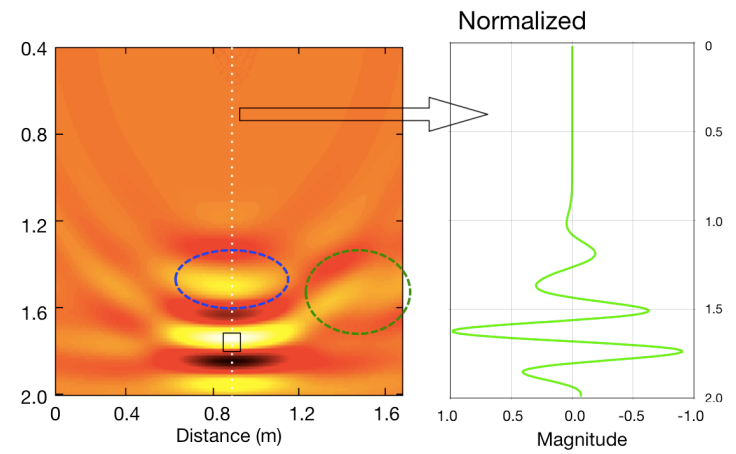

Figure 10: Subsurface image by a phased array antenna.

In this image, the undesired signals (blue and green dotted circles) are suppressed. We conclude that increasing the number of receivers is effective for the precise imaging of subsurface under surface structures.

\section{Conclusion}

Our results show that the phased array antenna has an advantage in enhancing the signal-to-noise ratio. Also, we confirmed that the phased array antenna enhanced the amplitude of scattered waves from the reflectors located out of the survey line. This property indicates the possibility to let us probe the subsurface under a structure such as buildings or tree-trunk. Moreover, we conclude that by controlling the directivity of EM waves in both phases of transmitting and receiving, we achieved the higher directivity in the GPR surveys.

We conclude that our phased array system has a potential to be used as an angular scanner imaging tool that has not yet been attempted in the conventional GPR system. This GPR system may reduce the number of survey lines and lower the cost of GPR surveys.

However, we confirmed that the undesired signals still remained in the observed signal. Those may induce a bad result in the migrated images. To overcome this problem, we are trying to introduce a Direction of Arrival method to our developing GPR.

\section{REFERENCES}

1) Hirano, Y., Dannoura, M., Aono, K., Igarashi, T., Ishii, M., Yamase, K., Makita, N., and Kanazawa, Y., 2009, Limiting factors in the detection of tree roots using ground-penetrating radar. Plant and Soil, 319, 15-24.

2) Lutz, P., and Perroud, H., 2006, Phased-array transmitters for GPR surveys. Journal of Geophysics and, Engineering, $3(1), 35$.

3) Hansen, Robert C, 2009, Phased array antennas, Wiley Series in Microwave and Optical Engineering, Vol.213, John Wiley \& Sons.

4) Uno, T., 1998, Simulations of EM fields and Antenna Analysis by FDTD method, Colona Sha, 1-57.

5) Yee, K. S., 1966, Numerical solution of initial boundary value problems involving Maxwell's equations in isotropic media, IEEE Trans. Antennas Propag., 14(3), 302-307.

6) Roden, J. A., \& Gedney, S. D., 2000, Convolutional PML (CPML): An efficient FDTD implementation of the CFS-PML for arbitrary media, Microwave and optical technology letters, 27(5), 334-338. 